\title{
MEMBRESÍA A BARRAS BRAVAS Y SU INFLUENCIA EN EL USO DE DROGAS
}

\author{
Catalina López-Quintero ${ }^{1, a}$, Yehuda Neumark ${ }^{1, b}$
}

\begin{abstract}
RESUMEN
Objetivos. Identificar los factores asociados con la membresía a las barras bravas, la incidencia del uso de drogas y la asociación entre membresía y uso de drogas. Materiales y métodos. Se analizan los datos reportados por 1303 adolescentes escolares que participaron en dos etapas (T1 y T2) de un estudio longitudinal realizado en 23 colegios de Bogotá, Colombia entre 2006 y 2007, seleccionados a partir de un muestreo probabilístico estratificado por conglomerados. Se implementaron modelos de regresión logística para estudiar las asociaciones de interés. Resultados. En T1, 8,2\% de los encuestados reportaron membresía a las barras bravas. Luego de un año de seguimiento, el $14,2 \%$ y el $4,6 \%$ de los estudiantes que afirmaron y negaron respectivamente pertenecer a una barra brava, iniciaron el consumo de drogas. La asociación entre membresía a las barras bravas e inicio del uso de drogas permanece después de ajustar por múltiples factores de confusión (RRA: 2,4; IC 95\%: 1,2 - 4,7). En el análisis estratificado por sexo, esta asociación permanece significativa únicamente en las mujeres (RRA: 6,1; IC 95\%: 2,5 - 15,3). Conclusiones. La membresía a las barras bravas mostró incrementar el riesgo de inicio del uso de drogas, particularmente en las mujeres. Los hallazgos implican que se deben focalizar los esfuerzos preventivos hacia las mujeres en dichos contextos.
\end{abstract}

Palabras clave: Abuso de drogas; Marihuana; Cocaína; Equipo deportivo; Fútbol; Vandalismo (fuente: DeCS BIREME).

\section{BARRAS BRAVAS MEMBERSHIP AND ITS INFLUENCE ON DRUG USE}

\begin{abstract}
Objectives. To identify the factors associated with barras bravas (soccer fans clubs) membership, the incidence in drug use and the association between membership and drug use. Materials and methods. This report analyzed data from 1303 adolescent scholars who participated in two stages (T1 and T2) of a longitudinal study done in 23 schools of Bogota during 2006- 2007, selected through a probabilistic sampling stratified by clusters. Logistic regression models were implemented in order to evaluate the associations of interest. Results. In T1, 8,2\% of the surveyed students reported barras bravas membership. After one year of follow-up, $14,2 \%$ of barras bravas members, and $4,6 \%$ of non-members, initiated drug use. The association between barras bravas membership and drug use onset remained significant after adjusting for multiple confounding factors $(\mathrm{ARR}=2,4 ; 95 \% \mathrm{Cl}=1,2-4,7)$. Sex-stratified analysis indicated that this association remained statistically significant only for females (ARR=6,1;95\% Cl=2,5-15,3). Conclusions. Barras bravas membership increases the risk of drug use onset, particularly among females. The findings imply that preventive efforts should be focused on females in these contexts.
\end{abstract}

Key words: Substance-Related Disorders; Marijuana; Cocaine; Sports equipment; Soccer; Vandalism (source: MeSH NLM).

\section{INTRODUCCIÓN}

Las barras bravas, torcidas, o porras son grupos organizados de seguidores de los equipos de fútbol que surgieron en la década de 1950 en Argentina y Uruguay, y que, posteriormente, se formaron en otros países de América Latina y los Estados Unidos ${ }^{(1)}$. Hoy se estima que existen alrededor de 250 barras bravas en todo el continente (2). En Colombia, las barras bravas aparecen en la década de 1990 y a lo largo de las últimas dos décadas estos grupos han protagonizado con frecuencia escándalos por actos de vandalismo, violencia, y uso y tráfico de drogas ${ }^{(1)}$.
Estudios sobre el comportamiento de los fanáticos del fútbol y otros deportes han logrado establecer una asociación entre la membresía a estos grupos y el consumo de sustancias psicoactivas ${ }^{(3-5)}$. Igualmente, se ha observado que el uso de estas sustancias en los miembros de estos grupos de fanáticos tiene como función facilitar los procesos de socialización, inclusión social, cohesión, y afirmación de roles masculinos ${ }^{(6)}$, lo que eventualmente puede significar la adopción de conductas de riesgo. Una dinámica similar se ha observado en las pandillas, en las cuales las sustancias psicoactivas cumplen una función fundamental en mantener la cohesión y estructura del grupo, facilitar la

Braun School of Public Health \& Community Medicine, Hebrew University-Hadassah. Jerusalen, Israel.

a Médico, doctora en Salud Pública; ${ }^{\mathrm{b}}$ Médico, doctora en Salud Pública y Epidemiología

Recibido: 17-01-12 Aprobado: 07-03-12 
adopción de conductas delictivas y comportamientos sexuales de alto riesgo así como la participación en actos de violencia dentro y contra otros grupos similares con el fin de establecer jerarquías y marcar territorialidad (7-9).

En Colombia, la literatura sobre las barras bravas tradicionalmente se ha centrado en establecer los aspectos socio-antropológicos de estos grupos y de sus expresiones de violencia ${ }^{(1,10-12)}$. A través de estos estudios se ha logrado determinar que la mayoría de sus integrantes son estudiantes o jóvenes con bajos ingresos económicos y pocas oportunidades laborales o de inclusión social, con altos niveles de impulsividad y agresividad, y que en su necesidad de buscar una identidad y reconocimiento social y dentro del grupo, terminan involucrándose en actos de violencia para crear zonas de dominio y control social (11). Asimismo, se ha logrado establecer que existen importantes intereses económicos en la formación y sostenimiento de las barras bravas, al constituirse estos grupos en una alternativa lucrativa para sus líderes, o al generar el poder suficiente para controlar mercados ilícitos.

Son escasos los estudios de carácter científico que evalúan la asociación entre la membresía a un grupo de fanáticos de deportes y el uso de drogas ${ }^{(4,6,13-15)}$, menos aún en el contexto latinoamericano o que empleen metodologías que evalúen la membresía a una barra brava como factor predictor en esta asociación. La presente investigación, a través del seguimiento a un grupo de escolares adolescentes en Bogotá (Colombia) por un periodo de doce meses busca identificar los factores asociados con la membresía a las barras bravas en hombres y mujeres, estimar la incidencia del uso de drogas según la membresía a barras bravas, y estimar la asociación entre membresía a barras bravas y uso de drogas en la población seleccionada para este estudio y según el sexo. El entendimiento de estos determinantes es crucial para el desarrollo de estrategias enfocadas a prevenir y reducir el consumo de drogas en los miembros de estos grupos y fortalecer el papel protector de estas redes sociales en los procesos de socialización de los jóvenes.

\section{MATERIALES Y MÉTODOS}

En este estudio longitudinal se seleccionó inicialmente una muestra de 2279 escolares provenientes de veintitrés colegios de Bogotá (nueve privados y catorce públicos) a través de un muestreo probabilístico estratificado multietápico por conglomerados. Los colegios fueron estratificados según su forma de financiación (pública o privada) y su estrato socio-económico (ESE) de acuerdo con lo establecido por la Secretaría de Educación de
Bogotá ${ }^{(16)}$. Se seleccionaron 66 clases de los grados octavos a décimo; en cada clase se invitó a participar a todos los estudiantes. En la primera etapa (T1), entre una población elegible de 2505 estudiantes, participaron 2361 estudiantes que asistieron a clase los días en que se aplicó la encuesta, contaron con la aprobación de sus padres y asintieron a participar en la encuesta (tasa de respuesta: 94,3\%). Posteriormente, se excluyeron 82 cuestionarios con información incompleta o incorrecta según los parámetros establecidos para validar los datos suministrados. De los 2279 estudiantes que proporcionan datos válidos en T1, se logró el seguimiento y apareamiento completo de los cuestionarios de 1592 estudiantes en la segunda etapa (T2) (tasa de abandono: $30,2 \%$ ). Con el fin de determinar la incidencia del uso de drogas se excluyeron los datos de 289 estudiantes que reportaron haber consumido drogas en T1 y que no reportaron membresía a las barras bravas. Finalmente para este estudio se analizaron los datos suministrados por 1303 estudiantes a quienes llamaremos "población del estudio".

\section{ENCUESTA}

Entre marzo y octubre de 2006 (T1) y entre marzo y octubre de 2007 (T2) se aplicó una encuesta estandarizada que incluía principalmente preguntas derivadas de la encuesta del comportamiento de riesgo de la juventud de los Estados Unidos ${ }^{(17)}$, el Inventario de Tamizaje sobre del Uso de Drogas ${ }^{(18)} \mathrm{y}$, en particular, el cuestionario utilizado en la investigación multinacional del proyecto PACARDO ${ }^{(19)}$. El cuestionario fue evaluado durante un estudio piloto que incluyó tres sesiones de grupos focales que permitieron valorar la idoneidad de las preguntas, el lenguaje, la comprensión de los constructos y el nivel de dificultad para completar el cuestionario en el tiempo asignado. Durante la aplicación del cuestionario, se solicitó a los maestros y el personal del colegio ausentarse de las aulas, se unificaron conceptos respecto a la definición de "droga" y un asistente de investigación leyó cada pregunta en voz alta y respondió a las preguntas de los estudiantes, todo esto con el fin de reducir al mínimo la probabilidad de sesgo de información, mejorar la confidencialidad y mantener el orden y decoro en las aulas. Con el fin de incrementar la confidencialidad y disminuir los sesgos de información, los cuestionarios de T1 y T2 fueron apareados a través de un código alfanumérico personal basado en un número limitado de caracteres (letra inicial del nombre, fecha de nacimiento y última letra del apellido) que cada estudiante generó. Se informó a los padres de familia acerca del objetivo del estudio, su contenido y los posibles riesgos asociados a la participación en el estudio según lo indica la ley 8430 de 1993 y se solicitó un consentimiento pasivo. Igualmente se solicitó el asentimiento de los estudiantes para participar en el 
estudio. El protocolo de investigación fue aprobado por el Comité de Investigación Universitario de la Facultad de Medicina de la Fundación Universitaria Juan N. Corpas en Colombia y de la Facultad de Medicina en la Universidad Hebrea en Israel.

\section{VARIABLES}

La incidencia de uso de drogas ilegales se definió operacionalmente al preguntar en $\mathrm{T} 2$ a los estudiantes ¿qué edad tenías cuando usaste por primera vez (nombre de la droga)? Las drogas evaluadas incluyen la marihuana, los inhalantes, la cocaína, el bazuco (un semiprocesado de pasta de coca mezclada con otros ingredientes) y el éxtasis.

La membresía a las barras bravas se determinó en T1 con una pregunta dicotómica que evaluaba la pertenecía a estos grupos (¿perteneces a una barra brava?). Las variables sociodemográficas evaluadas incluyeron: sexo, grupo de edad $(<14,14-16,>16$ años) y el ESE familiar. En Bogotá, el ESE familiar se determina de acuerdo con las condiciones de vida, incluyendo el tipo de construcción y materiales de las viviendas, y se utiliza para la codificación oficial de los barrios, y para establecer el monto de impuestos municipales y el costo de los servicios públicos. La ESE se define en seis categorías, siendo 1 el estrato más desfavorecido y 6 el más favorecido. Posteriormente, esta variable fue recodificada en ESE 1 y 2 (bajo), ESE 3 (medio) y ESE 4-6 (alto).

Partiendo de teorías de comportamiento usualmente empleadas para estudiar el uso de drogas en adolescentes ${ }^{(20)}$, se seleccionaron una serie de constructos psicosociales, que han mostrado influir en la incidencia del uso de drogas y que, eventualmente, podrían modificar o distorsionar la asociación en estudio ${ }^{(21-23)}$. Dichos constructos fueron evaluados en $\mathrm{T} 1 \mathrm{e}$ incluyeron: la percepción de riesgo, las actitudes frente al uso de drogas y la intención de uso de drogas. La percepción de riesgo se evaluó para cada sustancia psicoactiva con la pregunta ¿en qué medida crees que las personas se arriesgan a sufrir daño físico o psicológico, si utilizan (nombre de la sustancia psicoactiva) semanalmente?, con opciones de respuesta: ninguna/no sé, riesgo bajo, riesgo medio, y riesgo alto. Las respuestas "ninguno/ no sé " o "riesgo bajo" para una o más sustancias, se categorizaron como "baja percepción de riesgo", las opciones restantes como "alta percepción de riesgo". Las actitudes frente al uso de drogas ilegales se evaluaron con cinco preguntas dicotómicas que valoraban la necesidad de establecer leyes más estrictas contra el consumo de drogas y la incomodidad que producía a los estudiantes ver a la gente usando, ofreciendo o con curiosidad por usar drogas, o en estado de embria- guez (ejemplo: ¿las leyes en contra del uso de drogas deberían ser más estrictas? o ¿te molesta ver a la gente usando drogas?, con coeficiente alfa de Cronbach para la confiabilidad interna: 0,63 ). Una o más respuestas negativas fueron recodificadas como actitudes favorable al uso de drogas. La intención de usar alguna droga fue evaluada para cada sustancia con la pregunta: ¿qué tan probable es que uses (nombre de la droga) el próximo año? Las posibles respuestas incluían "improbable", "probable" y "muy probable". Respuestas "probable" o "muy probable" a por lo menos una droga se recodificaron como intenciones positivas al uso de drogas. El nivel de supervisión y control de los padres se determinó con seis preguntas dicotómicas que evaluaban el grado de envolvimiento de los padres en las actividades del adolescente, el conocimiento de las necesidades de sus hijos y estrategias de comunicación y crianza (por ejemplo, "¿tus padres o tutores normalmente saben dónde estás y lo que está haciendo?","¿tus padres o tutores normalmente saben que te gusta y que no te gusta?" con coeficiente alfa de Cronbach para la confiabilidad: $0,53)$. La escala acumulativa de supervisión y control de los padres fue recodificada en cuartiles como 10 alto nivel (0-3), 2 (4), 3 (5) y 4 o bajo nivel (6).

\section{ANÁLISIS ESTADÍSTICO}

Se calcularon estadísticas de primer orden con el fin de caracterizar la población del estudio y la población según su membresía a las barras bravas. Se implementaron modelos de regresión logística binaria con el fin de estudiar las asociaciones entre membresía a una barra brava y diferentes características sociodemográficas, individuales y del grupo familiar. Igualmente, se implementaron modelos de regresión logística bivariados y multivariados con el fin de evaluar la asociación entre la membresía a una barra brava en la incidencia de uso de drogas sin ajustar y ajustando por factores de confusión, respectivamente. Para esto, se desarrollaron tres modelos de regresión logística multivariados, el primero ajustando por las variables sexo, grupo de edad, estrato socioeconómico familiar, la percepción de riesgo, las actitudes frente al uso de drogas, la intención de uso de drogas en el próximo año, y la supervisión y control parental (modelo 1); el segundo con las variables incluidas en el modelo $1 \mathrm{y}$ adicionando el término de interacción entre sexo y membresía las barras bravas (modelo 2) y, el tercero, correspondiente al modelo 1 , excluyendo la variable sexo, y aplicado en hombres y en mujeres independientemente (modelo 3 ). Las asociaciones entre membresía a las barras bravas y las características establecidas en la línea de base se expresan como odds ratio $(\mathrm{OR})$ y odds ratio ajustado (ORA) con sus intervalos de confianza (IC) del 95\%, las asociaciones entre la membresía a las barras bravas y la 
incidencia del uso de drogas se expresan como riesgos relativos (RR) en los modelos univariados y como riesgo relativo ajustado (RRA) en los modelos multivariados. Las estimaciones del RRA se derivan de la fórmula de corrección de los odds ratio para estudios longitudinales propuesta por Zhang y Yu ${ }^{(24)}$. Como era de esperarse, con tasas de incidencia del uso de drogas inferiores al $10 \%$, los resultados de los modelos al aplicar la corrección fueron muy similares a los encontrados sin la corrección, salvo por intervalos de confianza menos amplios. Se definieron como diferencias estadísticamente significativas aquellas con valores de $p<0,05$ para todas las pruebas. Se evaluó la bondad del ajuste de los modelos de regresión logística mediante el test de Hosmer-Lemeshow, encontrándose ajuste adecuado en los modelos multivariados. Los análisis se realizaron utilizando el programa SPSS, versión 17.0 (SPSS Inc. Chicago, IL).

\section{RESULTADOS}

\section{CARACTERÍSTICAS DE LA POBLACIÓN EN ESTUDIO Y SEGÚN LA MEMBRESÍA A LAS BARRAS BRAVAS}

Las características de los estudiantes que no reportaron haber usado drogas en T1 se presentan en la Tabla 1.
Casi la mitad de este grupo era de sexo masculino, alrededor de dos tercios expresaron actitudes favorables hacia el uso de drogas (aproximadamente uno de cada ocho alumnos reportó una percepción de riesgo baja para el uso semanal de drogas) o intención de usar drogas el próximo año. El 8,2\% de los entrevistados reportó membresía a las barras bravas en T1, siendo significantemente mayor la proporción de hombres $(10,9 \%)$ que mujeres $(5,7 \%)(p<0,001)$.

\section{FACTORES ASOCIADOS A LA MEMBRESÍA A LAS BARRAS BRAVAS}

Comparado con sus contrapartes, los estudiantes con baja percepción de riesgo al uso semanal de drogas (OR: 2,9 ), actitudes favorables frente al uso de drogas (OR hombres: 2,8; OR mujeres: 2,0), intenciones de usar drogas en el próximo año (OR hombres: 3,0 ); y con niveles de control parental más bajo (4to cuartil, con relación al 1er cuartil, OR hombres: 3,2; OR mujeres: 4,3) reportaron una probabilidad significativamente mayor de pertenecer a una barra brava (Tabla 1).

\section{MEMBRESÍAA LAS BARRAS BRAVAS COMO FACTOR DE RIESGO PARA EL USO DE DROGAS}

El $14,2 \%$ de los estudiantes con membresía a las barras bravas reportó incidencia de uso de drogas después de

Tabla 1. Características sociodemográficas y variables relacionadas al uso de drogas de los participantes (miembros de barras bravas) que reportaron no usar drogas en T1. Bogotá, Colombia 2006.

\begin{tabular}{|c|c|c|c|c|c|c|c|}
\hline \multirow{2}{*}{ Características } & & \multirow{2}{*}{$\begin{array}{c}\text { Total de } \\
\text { participantes } \\
(n=1303) \\
n .^{\circ}(\%) \\
\end{array}$} & \multirow{2}{*}{$\begin{array}{c}\text { Total de } \\
\text { miembros } \\
(n=106) \\
n .{ }^{\circ}(\%)\end{array}$} & \multicolumn{2}{|c|}{$\begin{array}{l}\text { Hombres } \\
(n=66)\end{array}$} & \multicolumn{2}{|c|}{$\begin{array}{l}\text { Mujeres } \\
(\mathrm{n}=40)\end{array}$} \\
\hline & & & & $n .^{\circ}(\%)$ & OR (IC 95\%) & $n .^{\circ}(\%)$ & OR (IC 95\%) \\
\hline \multirow{3}{*}{ Edad (años) } & $<14$ & $200(15,3)$ & $14(13,2)$ & $10(15,2)$ & 1 & $4(10,0)$ & 1 \\
\hline & $14-16$ & $1039(79,7)$ & $83(78,3)$ & $49(74,2)$ & $0,9(0,5-1,9)$ & $34(85,0)$ & $1,7(0,6-4,9)$ \\
\hline & $>16$ & $64(5,0)$ & $9(8,5)$ & $7(10,6)$ & $1,9(0,7-5,5)$ & $2(5,0)$ & $2(0,4-11,7)$ \\
\hline \multirow[t]{3}{*}{ Estrato Socioeconómico familiar } & Bajo & $723(55,5)$ & $62(58,5)$ & $35(53,0)$ & & $27(67,5)$ & 1 \\
\hline & Medio & $404(31,0)$ & $32(30,2)$ & $22(33,3)$ & & $10(25,0)$ & $0,8(0,4-1,6)$ \\
\hline & Alto & $176(13,5)$ & $12(11,3)$ & $9(13,6)$ & & $3(7,5)$ & $0,5(0,2-1,7)$ \\
\hline $\begin{array}{l}\text { Baja percepción de riesgo del uso } \\
\text { semanal de drogas }\end{array}$ & & $159(12,2)$ & $28(26,4)$ & $18(28,6)$ & $2,9(1,6-5,3)$ & $10(26,3)$ & $2,9(1,3-6,2)$ \\
\hline $\begin{array}{l}\text { Actitudes favorables al uso de } \\
\text { drogas }\end{array}$ & & $778(59,7)$ & $82(77,4)$ & $53(81,5)$ & $2,8(1,5-5,4)$ & $29(72,5)$ & $2,0(1,0-4,1)$ \\
\hline $\begin{array}{l}\text { Intenciones de usar drogas el } \\
\text { próximo año }\end{array}$ & & $159(12,2)$ & $22(20,8)$ & $15(24,2)$ & $3,0(1,5-5,7)$ & $7(17,5)$ & $1,4(0,6-3,3)$ \\
\hline \multirow[t]{4}{*}{$\begin{array}{l}\text { Supervisión y control de los } \\
\text { padres (cuartiles) }^{a}\end{array}$} & 1 (alto) & $357(26,9)$ & $19(17,9)$ & $15(22,7)$ & 1 & $4(10,0)$ & 1 \\
\hline & 2 & $389(29,9)$ & $29(27,4)$ & $17(25,8)$ & $1,2,(0,6-2,5)$ & $12(30,0)$ & $2,5(0,8-7,8)$ \\
\hline & 3 & $272(20,9)$ & $19(17,9)$ & $11(16,7)$ & $1,1(0,5-2,4)$ & $8(20,0)$ & $2,4(0,7-8,2)$ \\
\hline & 4 (bajo) & $277(21,2)$ & $39(36,7)$ & $23(34,8)$ & $3,2(1,6-6,3)$ & $16(40,0)$ & $4,3(1,4-13,1)$ \\
\hline
\end{tabular}

OR: Odds ratio. ${ }^{a}$ Se excluyen los datos de ocho estudiantes que no reportaron información sobre el nivel de supervisión y control de los padres. 
Tabla 2. Uso de diferentes tipos de droga según membresía a las barras bravas, y según sexo. Bogotá, Colombia 2006-2007.

\begin{tabular}{lrrrrrrr}
\hline & $\mathrm{N}$ & $\begin{array}{c}\text { Marihuana } \\
\mathbf{n} .^{\circ}(\%)\end{array}$ & $\begin{array}{c}\text { Inhalantes } \\
\text { n. }{ }^{\circ}(\%)\end{array}$ & $\begin{array}{c}\text { Cocaína } \\
\text { n. }{ }^{\circ}(\%)\end{array}$ & $\begin{array}{c}\text { Bazuco } \\
\text { n. }{ }^{\circ}(\%)\end{array}$ & $\begin{array}{c}\text { Éxtasis } \\
\text { n. }{ }^{\circ}(\%)\end{array}$ & $\begin{array}{c}\text { Cualquier droga } \\
\text { n. }{ }^{\circ}(\%)\end{array}$ \\
\hline Miembros de barras bravas & 106 & $11(10,4)$ & $3(2,8)$ & $1(0,9)$ & $1(0,9)$ & $2(1,9)$ & $15(14,2)$ \\
$\quad$ Hombres & 66 & $5(7,6)$ & $1(1,5)$ & $1(2,5)$ & 0 & 0 & $6(9,1)$ \\
$\quad$ Mujeres & 40 & $6(15,0)$ & $2(5,0)$ & 0 & $1(2,5)$ & $2(5,0)$ & $9(22,5)$ \\
No miembros de barras bravas & 1197 & $29(2,4)$ & $14(1,2)$ & $11(0,9)$ & $4(0,3)$ & $11(0,9)$ & $55(4,6)$ \\
$\quad$ Hombres & 537 & $17(3,2)$ & $9(1,7)$ & $7(1,3)$ & $3(0,6)$ & $6(1,1)$ & $31(5,8)$ \\
$\quad$ Mujeres & 660 & $12(1,8)$ & $5(0,8)$ & $4(0,6)$ & $1(0,2)$ & $5(0,8)$ & $24(3,6)$ \\
\hline
\end{tabular}

12 meses de seguimiento. Este porcentaje fue significativamente menor en los estudiantes que no reportaron membresía en las barras bravas $(4,6 \%)(p<0,001)$. La marihuana fue la sustancia usad con más frecuencia $(10,3 \%)$, seguido de los inhalantes $(2,8 \%)$, el éxtasis $(1,9 \%)$, la cocaína $(0,9 \%)$ y el bazuco $(0,9 \%)$. Aunque se encontraron diferencias por sexo, ninguna de estas diferencias demostró ser significativa (Tabla 2).

Después de ajustar por sexo, grupo de edad, estrato socioeconómico familiar, la percepción de riesgo, las actitudes frente al uso de drogas, la intención de uso de drogas en el próximo año, y la supervisión y control parental (Modelo 1), los estudiantes que reportaron membresía en las barras bravas durante $\mathrm{T} 1$, tuvieron una probabilidad dos veces mayor que los que no reportaron membresía a iniciar el uso de drogas (RRA: 2,4; IC95\%: 1,2-4,7). Sin embargo, al incluir la interacción entre membresía a las barras bravas y sexo en el modelo (Modelo 2) esta asociación desaparece (RRA: 0,2; IC95\%: 0,1-1,6) y se hace evidente la interacción entre membresía a las barras bravas y sexo femenino (RRA: 6,0; IC95\%: 1,5-23,4). Posteriormente, el análisis estratificado por sexo indica que la asociación entre membresía a las barras bravas e incidencia del uso de drogas fue estadísticamente significativa en mujeres (RRA: 6,1; IC95\%: 2,4-15,2) pero no en hombres (RRA: 0,9; IC $95 \%$ : 0,3-2,5).

\section{DISCUSIÓN}

Este primer estudio sobre la membresía a barras bravas y su relación con el uso de drogas permitió evidenciar que 1) uno de cada doce estudiantes en Bogotá tiene membresía en las barras bravas, 2) la membresía a las barras bravas está asociada con la baja percepción de riesgo al uso frecuente de drogas, las actitudes favorables frente al uso de drogas, las intenciones de usar drogas en el próximo año y con niveles bajos de control parental, 3) la membresía a las barras bravas predice el inicio del consumo de drogas después de un año de seguimiento en las mujeres.

La membresía a las barras bravas está asociada con actitudes, creencias o intenciones que favorecen el uso de drogas tanto en hombres, como en mujeres. Aunque no es posible establecer la direccionalidad de la asociación, es claro que se necesitan intervenciones preventivas dentro de estos grupos, que permitan modificar la percepción de riesgo frente al uso frecuente de drogas $\mathrm{y}$ las actitudes e intenciones de consumo, y que consecuentemente disminuyan la tendencia de estos jóvenes a involucrarse en comportamientos de riesgo.

Expendedores de drogas y organizaciones criminales han logrado permear de manera efectiva estos grupos de fanáticos e inducir el consumo de drogas en menores que ingresan a las barras bravas motivados por su afición al futbol y su necesidad de construir una identidad y socializar ${ }^{(1-5)}$. Las autoridades gubernamentales y las instituciones encargadas de organizar los campeonato profesionales se han limitado a diseñar leyes que garanticen la seguridad en los escenarios deportivos (Decreto 164 de 2004, Acuerdo 390 de 2005, Acuerdo 062 de 2006 y Ley 4040 de 2007) e implementar intervenciones policiales y pedagógicas de corto plazo para reducir los actos de violencia y vandalismo en los estadios, sin entender y atender el origen de la problemática en los individuos con membresía en estos grupos. Igualmente, ha sido muy limitada la participación de los clubes en el desarrollo de dichos procesos y en intentar restituir la deteriorada imagen de sus seguidores. La redefinición de la identidad colectiva de las barras bravas requiere de intervenciones encaminadas a reconocer su función dentro de los procesos de socialización y formación de identidad en los jóvenes fanáticos ${ }^{(25)}$, y su potencial para promover estilos de vida saludables. Dichas intervenciones deben desarrollarse de manera continua y estructurada e imprescindiblemente involucrar a la familia, los colegios, a las instituciones deportivas directamente vinculadas a la organización de los campeonatos y a los clubes deportivos. 
Curiosamente, la asociación entre membresía a las barras bravas e incidencia en el inicio en el uso de drogas fue significativa únicamente en las mujeres. Previos estudios han logrado establecer que independientemente del sexo biológico la membresía en grupos de fanáticos a los deportes está correlacionada con la orientación de género masculino ${ }^{(6,26)}$. Partiendo de estos hallazgos y de la alta correlación entre la construcción de identidad masculina y la adopción de comportamientos impulsivos y conductas de riesgo, es posible explicar la tendencia incrementada en las mujeres con membresía a las barras bravas a consumir drogas con relación a aquellas que reportaron no pertenecer a estos grupos. Por otro lado, las barras bravas podrían facilitar la disponibilidad de las drogas e incrementar las oportunidades de uso no solo por permitir el fácil acceso a las sustancia, sino también por ofrecer una "justificación" para el consumo, como puede ser los rituales de celebración de los logros del equipo. La identificación de las mujeres como un subgrupo de mayor riesgo dentro de las barras bravas, permite orientar los esfuerzos hacia el desarrollo de estrategias de prevenciones selectivas, sensibles a los procesos de desarrollo biológico, cognitivo y conductual de las mujeres.

La interpretación de los hallazgos descritos en este estudio requiere tener en cuenta algunas limitaciones. Usualmente se reportan con menor frecuencia los comportamientos no normativos, por lo cual es común observar en los estudios epidemiológicos de uso de drogas que se subestiman la prevalencia o las tasas de incidencia de uso de drogas ${ }^{(27)}$. Los resultados del estudio únicamente pueden generalizarse a la población escolar de Bogotá. Al aplicar la encuesta en escuelas, se excluyeron adolescentes no escolarizados, quienes probablemente presentan una mayor prevalencia de conductas de riesgo que la población escolarizada ${ }^{(28)}$ lo que podría ocasionar una subestimación de las tasas de incidencia de uso de drogas. Igualmente, el abandono del $30 \%$ de los estudiantes que participaron en T1 los cuales mostraron una mayor prevalencia de factores de riesgo, pudo eventualmente ocasionar subestimación de dichas tasas. La confiabilidad de las constructos evaluados en este estudio fue menor a la reportada en el estudio de PACARDO, por ejemplo se encontró un coeficiente alfa de Cronbach de 0,53 para la escala de supervisión y control de los padres en este estudio, en comparación con 0,70 observado en el estudio de PACARDO ${ }^{(19)}$. La confiabilidad moderada de los coeficientes observados en este estudio pone en evidencia la necesidad de validar las escalas localmente, ya que diferencias culturales y sociales pueden influir en la manera en que estos constructos operan. La determinación de la membresía a las barras bravas con una sola pregunta limita evaluar el grado de participación en las actividades de las barras y, en consecuencia, el impacto real de la membresía en estos grupos en la adopción de comportamientos de riesgo. A pesar de estas limitaciones, este primer estudio longitudinal de la incidencia de uso de drogas según la membresía a las barras bravas en Bogotá, amplía el nivel actual de conocimientos sobre la epidemiología del consumo de drogas y proporciona información oportuna para el diseño de programas preventivos.

Los hallazgos de este estudio sugieren que se requieren estudios cualitativos y cuantitativos que permitan identificar detalladamente los mecanismos psicosociales que conducen al inicio y progresión en el consumo de drogas en las mujeres con membresía en barras bravas. Así mismo, es necesario documentar a través de estudios futuros la relación entre uso de drogas y crimen en las barras bravas, con el fin de poder establecer de manera más precisa el impacto social y económico de este problema.

El desarrollo de estrategias preventivas encaminadas a reducir los comportamientos problemáticos en los miembros de las barras bravas requiere la participación de actores en múltiples sectores. Estrategias como el programa de acreditación de clubes Good Sports ${ }^{(29,30)}$ parecen ser prometedoras en reducir el consumo de alcohol en los miembros de los clubes deportivos, y pueden ser eventualmente replicadas en Latinoamérica, una vez se adapten y establezca su eficacia a través de ensayos aleatorios controlados.

\section{Contribuciones de autoría}

CLQ y YN participaron en el diseño del estudio, el análisis de los datos, revisaron críticamente el artículo y aprobaron la versión final.

\section{Fuentes de financiamiento}

Beca doctoral Milstein a Catalina Lopez-Quintero.

\section{Conflictos de interés}

Los autores declaran no tener conflictos de interés en la publicación de este artículo.

\section{REFERENCIAS BIBLIOGRÁFICAS}

1. Gómez G. La Violencia en el futbol, vista a través del fenómeno de las barras bravas [Tesis de Licenciatura]. Bogotá, DC: Facultad de Ciencias Humanas, Universidad Nacional de Colombia; 2001.

2. Barrabrava.net [Internet]. c2009-2012 [citado el 7 de marzo, 2012]. Disponible en: http://barrabrava.net/historia/historia_del_movimiento_barra_brava.html

3. Glassman T, Werch CE, Jobli E, Bian H. Alcohol-related fan behavior on college football game day. J Am Coll Health. 2007;56(3):255-60.

4. Merlo LJ, Ahmedani BK, Barondess DA, Bohnert KM, Gold MS. Alcohol consumption associated with collegiate 
American football pre-game festivities. Drug Alcohol Depend. 2011;116(1-3):242-5.

5. Mattick AP, Mehta R, Hanrahan H, O'Donnell JJ. The Football World Cup 2002--analysis of related attendances to an Irish Emergency Department. Ir Med J. 2003;96(3):90-1.

6. Ayres TC, Treadwell J. Bars, drugs and football thugs: Alcohol, cocaine use and violence in the night time economy among English football firms. Crime Crim Justice. 2012;12(1):83 -100.

7. Cepeda A, Valdez A. Risk behaviors among young mexican american gang-associated females: sexual relations, partying, substance use, and crime. J Adol Res. 2003;18(1):90106.

8. Harper GW, Robinson WL. Pathways to risk among inner-city African-American adolescent females: The influence of gang membership. Am J community Psycho. 1999;27(3):383-404.

9. Hunt GP, Laidler KJ. Alcohol and violence in the lives of gang members. Alcohol Res Health. 2001;25(1):66-71.

10. Bernal A, Rivera O. Memorias urbanas y archivos transeúntes en el fenómeno de las barras bravas en Bogotá. Encuentro Internacional de Historia Oral "Oralidad y Archivo de la Memoria”. Mayo 5, 6 y 7 de 2005. Bogotá: Colectivo de Historia Oral y Asociación Pedagógica "APPTOS"; 2005.

11. Clavijo J. Estudio de barras de fútbol de Bogotá: Los comandos azules. Universitas Humanística. 2004;31(58):42-59.

12. Dávila $A$, Londoño $C$. La nación bajo un uniforme. Fútbol $e$ identidad nacional en Colombia 1985-2000. En: Alabarces $\mathrm{P}$, compilador. Futbologías: Fútbol, identidad y violencia en América Latina. $1^{\mathrm{a}}$ ed. Buenos Aires: Clacso; 2003.

13. Nelson TF, Wechsler H. School spirits: Alcohol and collegiate sports fans. Addict Behav. 2003;28(1):1-11.

14. Wolfe J, Martinez R, Scott WA. Baseball and beer: An analysis of alcohol consumption patterns among male spectators at major-league sporting events. Ann Emerg Med. 1998;31(5):629-32.

15. Di Pietro MC, Doering-Silveira EB, Oliveira MP, Rosa-Oliveira LQ, Da Silveira DX. Factors associated with the use of solvents and cannabis by medical students. Addict Behav. 2007;32(8):1740-4

16. Secretaría de Educación del Distrito Capital [Internet]. Bogotá: Secretaría de Educación del Distrito Capital; c2012 [actualizado el 4 de octubre de 2011; citado el 12 de diciembre de 2011]. Disponible en: http://www.sedbogota.edu.co/

17. Tarter RE. Evaluation and treatment of adolescent substance abuse: a decision tree method. Am J Drug Alcohol Abuse. 1990;16(1-2):1-46.

18. Centers for Disease Control and Prevention (CDC). Handbook for conducting youth risk behavior surveys. Atlanta, GA: US Department of Health and Human Services, CDC, National Center for Chronic Disease Prevention and Health Promotion; 2003.

19. Dormitzer CM, Gonzalez GB, Penna M, Bejarano J, Obando P, Sanchez M, et al. The PACARDO research project: Youthful drug involvement in Central America and the Dominican Republic. Rev Panam Salud Publica. 2004;15(6):400-16.
20. Ajzen I, Fishbein, M. Attitudes and the attitude-behavior relation: Reasoned and automatic processes. En: Stroebe W, Hewstone M, ed. Review of Social Psychology. Chichester: John Wiley \& Sons; 2000. P. 1-33.

21. Brook JS, Brook DW, De La Rosa M, Whiteman M, Montoya ID. The role of parents in protecting Colombian adolescents from delinquency and marijuana use. Arch Pediatr Adolesc Med. 1999;153(5):457-64.

22. Ellickson PL, Martino SC, Collins RL. Marijuana use from adolescence to young adulthood: Multiple developmental trajectories and their associated outcomes. Health Psychol. 2004;23(3):299-307.

23. Guxens M, Nebot M, Ariza C, Ochoa D. Factors associated with the onset of cannabis use: A systematic review of cohort studies. Gac Sanit. 2007;21(3):252-60.

24. Zhang J, Yu KF. What's the relative risk? A method of correcting the odds ratio in cohort studies of common outcomes. JAMA. 1998; 280(19):1690-1.

25. Brown WJ, de Matviuk MA. Sports celebrities and public health: Diego Maradona's influence on drug use prevention. J Health Commun. 2010;15(4):358-73.

26. Wann DL, Waddill PJ, Dunham MD. Using sex and gender role orientation to predict level of sport fandom. J Sport Behav. 2002;27:367-77.

27. Anthony J, Neumark Y, ML VE. Do I do what I say? A perspective on self-report methods in drug dependence epidemiology. En: Stone A, Turkan, JS, Bachrach CA, Jobe JB, Kurtzman HS, Cain VS (Eds.). The Science of Self-Report: Implications for Research and Practice. New.Jersey: Lawrence Erlbaum Associates; 2000. p. 175-98.

28. Instituto Colombiano de Bienestar Familiar (ICBF), Dirección Nacional de Estupefacientes (DNE). Estudio nacional de consumo de sustancias psicoactivas en adolescentes en conflicto con la ley en Colombia. Bogotá: ICBF/DNE; 2009.

29. Kingsland M, Wolfenden L, Rowland BC, Tindall J, Gillham $\mathrm{KE}, \mathrm{McElduff} \mathrm{P}$, et al. A cluster randomised controlled trial of a comprehensive accreditation intervention to reduce alcohol consumption at community sports clubs: study protocol. BMJ Open. 2011;1(2):bmjopen2011000328.

30. Rowland B, Allen F, Toumbourou JW. Impact of alcohol harm reduction strategies in community sports clubs: $\mathrm{Pi}$ lot evaluation of the good sports program. Health Psychol. 2011 Dec 12. [Epub ahead of print].

Correspondencia: Catalina López-Quintero

Dirección: Braun School of Public Health \& Community Medicine, Hebrew University-Hadassah, P.O.B. 12272, Jerusalem 91120, Israel

Teléfono: 972-2-6439103 / 972-2-6431086

Correo electrónico: catalina.lopez@mail.huji.ac.il 